\title{
Nanostructured Molecularly Imprinted Polyaniline for Acrylamide Sensing ${ }^{\dagger}$
}

\author{
Anja Drame ${ }^{1,2, *}$, Špela Trafela ${ }^{1,2}$ and Kristina Žužek Rožman ${ }^{1,2}$ \\ 1 Department for Nanostructured Materials, Jožef Stefan Institute, Jamova 39, Si-1000 Ljubljana, Slovenia \\ 2 Jožef Stefan International Postgraduate School, Jamova 39, Si-1000 Ljubljana, Slovenia \\ * Correspondence: anja.drame@ijs.si or anja.korent@ijs.si \\ + Presented at the 7th International Symposium on Sensor Science, Napoli, Italy, 9-11 May 2019.
}

Published: 13 August 2019

\begin{abstract}
Due to the non-electroactivity of carcinogenic organic compound acrylamide, an indirect electrochemical detection by using molecularly imprinted polymer is proposed as a detection method. Polyaniline was chosen due to the conductivity, interesting properties and wide usage in the field of sensors. Polyaniline was prepared by the different electropolymerisation process to achieve different morphologies, properties. Cyclic voltammetry was further chosen for electropolymeristion of polyaniline, in which propanamide was imprinted as a template molecule. Due to the same size, shape and functional groups, so imprinted polyaniline presents a proof of concept molecule for further acrylamide bonding. Successful imprinting was confirmed by surface analysis technique (FTIR) and simple electrochemical measurements of polyaniline response.
\end{abstract}

Keywords: acrylamide; molecularly imprinted polymer; polyaniline; screen-printed electrode; sensing

\section{Introduction}

Acrylamide (AA), $\mathrm{C}_{3} \mathrm{H}_{5} \mathrm{NO}$, is a colourless, non-volatile, water-soluble organic compound with wide industrial use. Naturally is formed in heat-treated food [1] via Maillard reaction between amino acid (asparagine) and a carbonyl group of a reducing sugar at above $120^{\circ} \mathrm{C}$. As AA is a neurotoxic and genotoxic compound, it was in 1994 classified as a potential carcinogen to humans (Group 2A) [1]. From 2018 a new EU regulation requires that food business operators (FBOs) apply mandatory measures to reduce the presence of AA [2]. Until now, the most frequently used methods for detection of AA are based on gas chromatography (GC), high-performance liquid chromatography (HPLC), mass spectrometry (MS), and the combination of these. However, all the mentioned techniques are expensive, time-consuming, and they require a qualified operator and a sample preparation. On the other hand, electrochemical sensors show great potential due to their low cost, small size, fast response, portability, good sensibility, selectivity, low detection limit and are user-friendly [3,4]. Up to date, electrochemical AA sensors are based on molecular imprinting of nonconductive polymer which limits their performance because of probe molecules that have to be used.

Molecularly imprinted polymers (MIP) are synthetic polymers with specific recognition sites for targeted molecule based on a monomer, template and a cross-linker. Several nonconductive functional monomers [3-6]) were already used for the creation of MIP for AA molecule. Two of them $[5,6]$ were used just for adsorption of AA for its indirect determination with HPLC. And the other two [3,4] were used for electrochemical determination of AA concentration in aqueous samples by using additional redox probe molecule - potassium ferricyanide or potassium ferrocyanide.

Conductive polymer polyaniline (PANI) has many advantages in sensor applications such as simple synthesis, possibility of deposition on various substrates $(\mathrm{Pt}, \mathrm{Au}$, ITO, graphite, glassy carbon etc.), diversity of structural forms (nanoparticles or 1-D fibres), high ambient environmental stability, 
unique electrochemical properties and low cost [7,8]. PANI is electrochemically deposited by anodic electropolymerisation (oxidative polymerisation) [9]. Due to the oxidation/reduction state, PANI exists in three forms: leucoemeraldine (fully reduced), emeraldine (half oxidised) and pernigraniline (fully oxidised). During electropolymerisation in an acidic solvent, polyaniline is protonated. These forms of PANI are conductive salts, and the most conductive form of PANI salt is emeraldine salt [8]. PANI is widely used as a material for ammonia detection (change in PANI properties due to the presence of amine group) [10] and as a functional monomer in MIP for detection of ascorbic acid [11]. Because of its potential, it will be investigated and used as a base material for a direct AA determination, without the presence of an additional redox probe.

\section{Materials and Methods}

\subsection{Characterisation of Screen-Printed Electrodes (SPE)}

Commercial screen-printed electrodes with the gold working electrode (Au-SPE), platinum counter electrode and silver reference electrode were purchased from Spanish DropSens company [12]. Electrodes were characterized by JEOL JSM 7600F scanning electron microscope (SEM) combined with energy dispersive $\mathrm{X}$-ray spectroscopy and profilometer to observe the morphology, chemical composition and surface profile.

\subsection{Synthesis and Characterisation of Polyaniline (PANI) on Au-SPE}

Deposition of PANI on Au-SPE was done by electropolymerisation of aniline monomer (0.1 M) in the acidic electrolyte $(1 \mathrm{M} \mathrm{HCl})$ using electrochemical techniques (cyclic voltammetry, chronopotentiometry and chronoamperometry) by PalmSens4 potentiostat. First successful depositions were studied by Perkin Elmer FT-IR spectrometer Fronteir L1280018, and the most appropriate procedure was chosen for further work. By varying polymerisation time, different stages of PANI formation were obtained. Morphology of obtained films was observed SEM.

\subsection{Preparation and Verification of Propanamide (PAM) Imprinted Polyaniline}

Deposition of imprinted PANI was obtained by using the same procedure as described above, just PAM as a template molecule was added to polymerization suspension. Verification of the presence of template was done by FTIR analysis and electrochemical measurements (cyclic voltammetry) in the acidic electrolyte $(10 \mathrm{mM} \mathrm{HCl})$.

\section{Results and Discussion}

Polyaniline (PANI) nanofibres/nanoparticles prepared by electropolymerisation in acidic solution were studied as a potential base material for molecularly imprinted polymer (MIP) system for acrylamide (AA) detection. The polymerisation was performed by electrochemical techniques in $\mathrm{HCl}$ on the commercial screen-printed electrode (SPE) with the gold working electrode, platinum counter electrode and silver reference electrode. PANI formation was studied on two different gold SPE electrodes which differ in the process of curing the gold ink (AT and BT electrode). According to SEM analysis, electrodes have different surface morphology, one is more porous and flat (AT), meanwhile other is composed of around micron big particles (BT). According to the SEM results of deposited PANI film by cyclic voltammetry (Figure 1, left), PANI deposited of AT electrode has the morphology of thinner fibres, meanwhile on BT electrode are PANI fibres are more concise (Figure 1). 

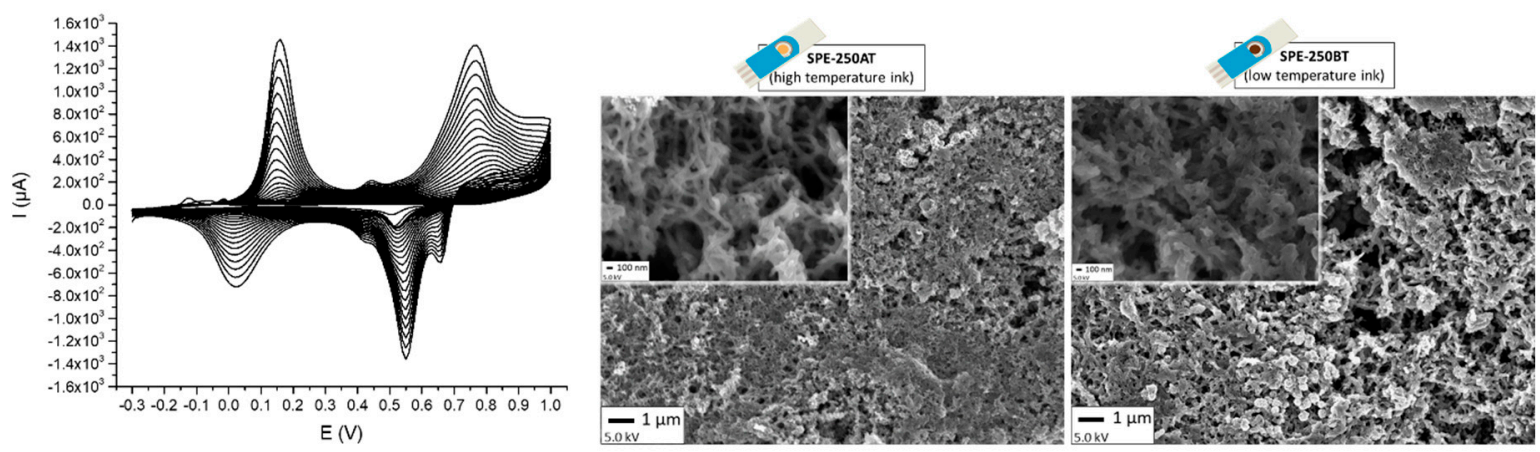

Figure 1. Morphology of PANI film deposited by cyclic voltammetry on two different commercial Au-SPE electrodes.

Deposition of polyaniline was confirmed by its characteristic peaks obtained by FTIR (Figure 2) and by the observed characteristic green colour of polymerised working electrode, which indicates emeraldine salt. Cyclic voltammogram (Figure 1, left) of electropolymerisation shows four main distinguish peaks, characteristic for polyaniline (1st oxidation peak: leucoemeraldine to emeraldine, $2^{\text {nd }}$ oxidation peak: emeraldine to pernigraniline and two reduction peaks for reverse transformation). Polymerisation stages of PANI formation were observed by stopping its polymerisation at certain hight of first oxidation peak (final current of peak) in cyclic voltammetry. At the beginning stages of polymerisation, PANI forms a film on the gold electrode which later transforms to a nanofibre-based structure. By analysing one of the most characteristic peaks for PANI formation (first oxidation peak at around $0.2 \mathrm{~V}$-the transition between leucoemeraldine to emeraldine salt), the mass and the film thickness was calculated by using Faraday's law. At the same final current $(1.5 \mathrm{~mA})$ of first oxidation peak (around 0.2 V), AT electrode showed to have higher amount of PANI deposited on surface (1.48 $\mu \mathrm{g})$ and was consequently covered with a thicker PANI film $(84.12 \mathrm{~cm})$ in comparison to BT electrode $(1.277 \mu \mathrm{g}$ and $72.59 \mathrm{~nm})$. Due to the better repeatability, AT electrode was chosen for further experiments.

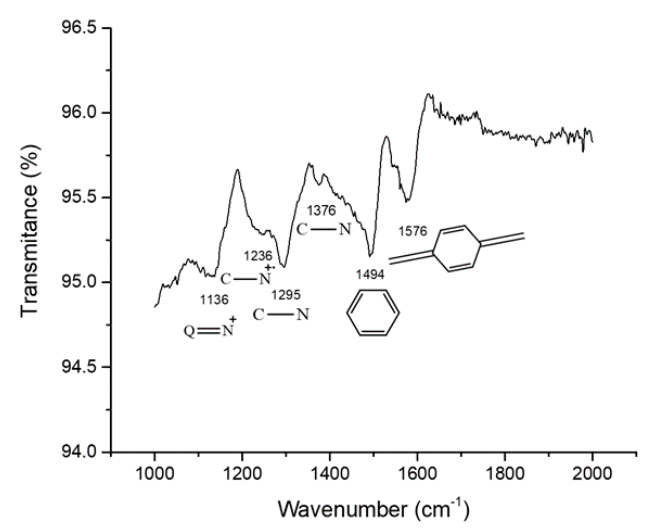

Figure 2. FTIR results of deposited PANI film on Au-SPE.

Imprinted PANI was prepared by adding different concentrations of template moleculepropanamide (PAM-structural analogue to acrylamide) into starting polymerisation suspension. FTIR results (Figure 3a) shows two new peaks in the range of 2849 and $3336 \mathrm{~cm}^{-1}$, characteristic for alkanes and amide group, which confirms successful imprinting of PAM. All other peaks are a consequence of the subtracting of just PANI electrode as a background. 

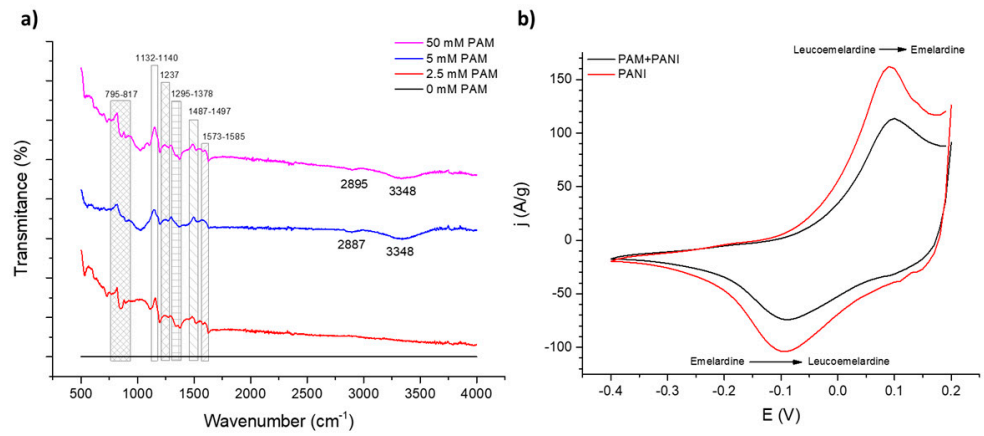

Figure 3. (a) FTIR results for PAM imprinted PANI using different PAM concentrations; (b) electrochemical conformation of imprinted PANI by 5 mM PAM.

Polyaniline has reversible redox pair (leucoemeraldine/emeraldine). In the presence of imprinted sites in PANI film (presence of unconducive PAM), the current response for the mentioned reversible reaction are lower in comparison to pure PANI and confirms successful imprinting.

\section{Conclusions}

Polyaniline was successfully electropolymerized onto different gold-based SPE electrodes by using electrochemical approach. It was prepared as conductive emeraldine salt which enables further usage in studies for designing molecularly imprinted sensing systems. By the addition of propanamide as a template molecule into polymerisation suspension, polyaniline polymerised around the template, causing its imprint. Obtained systems, after the template molecule removal, present the first stage in assembly of the acrylamide-sensitive receptor element. Further experiments will include studying of template extraction and techniques for monitoring its presence or absence. Our goal is to avoid usage of a redox probe. Therefore, our work will continue observing PANI reversible couple (leucoemeraldine/emeraldine) by using either cyclic voltammetry, chronoamperometry or electrochemical impedance spectroscopy.

Funding: This work was supported by Slovenian Research Agency (ARRS) [PR-08337].

\section{References}

1. Friedman, M. Chemistry, biochemistry, and safety of acrylamide. A review. J. Agric. Food Chem. 2003, 51, 4504-4526.

2. European Commission. Commission recommendation on establishing mitigation measures and benchmark levels for reduction of the presence of AA in food. Off. J. Eur. Union 2017, L304, $24-44$.

3. Liu, X., et al. Electrochemical Sensor based on Imprinted Sol-Gel Polymer on Au NPs-MWCNTs-CS Modified Electrode for the Determination of Acrylamide. Food analytical methods 2016, 9, 114-121.

4. Wang, Q., et al. An electrochemical sensor based on molecularly imprinted membranes on a P-ATP-AuNP modified electrode for the determination of acrylamide. Analytical Methods 2014, 6, 6452-6458.

5. Ji, J.; Sun, X.; Tian, X.; Li, Z.; Zhang, Y. Synthesis of acrylamide molecularly imprinted polymers immobilized on graphite oxide through Surface-Initiated atom transfer radical polymerization. Anal. Lett. 2013, 46, 969-981.

6. Jiang, D., X. Sun, and Y. Zhang, Preparation and application of acrylamide molecularly imprinted composite solid-phase extraction materials. Anal. Methods 2012, 4, 3760-3766.

7. Kaur, G., et al. Electrically conductive polymers and composites for biomedical applications. RSC Adv. 2015, 5, 37553-37567.

8. MacDiarmid, A.G. and Epstein, A.J. Polyanilines: A novel class of conducting polymers. Faraday Discuss. Chem. Soc. 1989, 88, 317-332.

9. Bavane, R.G. Synthesis and Characterization of Thin Films of Conducting Polymers for Gas Sensing Applications. Available online: https://shodhganga.inflibnet.ac.in/bitstream/10603/48043/7/07_table.pdf (accessed on 1 December 2018). 
10. Mikhaylov, S.; Ogurtsov, N.; Noskov, Y.; Redon, N.; Coddeville, P.; Wojkiewicz, J.-L.; Pud, A. Ammonia/amine electronic gas sensors based on hybrid polyaniline-TiO 2 nanocomposites. The effects of titania and the surface active doping acid. RSC Adv. 2015, 5, 20218-20226.

11. Roy, A.K.; Dhand, C.; Malhotra, B.D. Molecularly imprinted polyaniline film for ascorbic acid detection. J. Mol. Recognit. 2011, 24, 700-706.

12. DropSens. Screen-Printed Gold Electrodes. Available from: http://www.dropsens.com/en/pdfs_productos/ new_brochures/250at-250bt.pdf. (accessed on 18 December 2018).

(C) 2019 by the authors. Licensee MDPI, Basel, Switzerland. This article is an open access article distributed under the terms and conditions of the Creative Commons Attribution (CC BY) license (http://creativecommons.org/licenses/by/4.0/). 